\title{
Safety and Feasibility of the Venous Access via Internal Jugular Vein Puncture Approach for Totally Implantable Venous Access Device Placements Compared with Subclavian Vein Puncture
}

\author{
Shinichiro Koketsu ${ }^{1}$, Shinichi Sameshima ${ }^{1}$, Yawara Kubota ${ }^{1}$, Kosuke Hirano ${ }^{1}$, Asami Suzuki ${ }^{1}$, \\ Nana Makino ${ }^{1}$, Yoshitake Sugamata ${ }^{1}$, Hidemaro Yoshiba ${ }^{1}$, Takanori Kakihara ${ }^{2}$, Miwako Nozaki ${ }^{2}$, \\ Masatoshi Ooya ${ }^{1}$ \\ ${ }^{1}$ Department of Surgery, Koshigaya Hospital, Dokkyo Medical University, Koshigaya, Japan; ${ }^{2}$ Department of Radiology, Koshigaya \\ Hospital, Dokkyo Medical University, Koshigaya, Japan. \\ Email: koketsu-tky@umin.ac.jp
}

Received October $18^{\text {th }}, 2012$; revised November $20^{\text {th }}, 2012$; accepted November $28^{\text {th }}, 2012$

\begin{abstract}
Background: The aim of this study was to evaluate the safety and feasibility of venous access via the internal jugular vein (IJV) for totally implantable venous access device (TIVAD) placements. In Japan, TIVADs are generally placed in position by the percutaneous subclavian vein puncture approach (SVPA). However, this approach causes infrequent intraoperative or postoperative complications. Using the internal jugular vein puncture approach (IJVPA), TIVADs could be placed more easily and safely. Materials and Methods: Fifty-six patients who received TIVADs for chemotherapy of colorectal carcinomas were enrolled in this study. The choice of approach (IJVPA or SVPA) was adopted at the discretion of each doctor in charge of the patient. The operation time, success rate and complications of the two approaches were compared and evaluated. Results: TIVAD placement was successful in all patients. Thirty patients received the device via IJV puncture, but 1 patient required conversion to SVPA. Twenty-six patients underwent SVPA for device placement, but 3 of these patients required conversion to IJVPA. Mean operation time was 34.3 min in IJVPA and $35.2 \mathrm{~min}$ in SVPA. The success rate was $96.6 \%$ in IJVPA and $88.5 \%$ in SVPA. No severe perioperative complications were observed. However, long-term complications were observed in five cases, 3 by IJVPA and 2 by SVPA, but no significant difference in the rate of complications was observed between these two approaches. A catheter-related thrombosis was found by CT scan in 3 patients, two of whom underwent IJVPA (6.7\%) and one case underwent SVPA (3.8\%). Two patients received simultaneous administration of bevacizumab. Catheter infections occurred in 1 patient who underwent IJVPA (3.3\%) and 1 patient who underwent SVPA (3.8\%). Conclusions: The IJVPA is a safe and feasible method for TIVAD placement.
\end{abstract}

Keywords: Totally Implantable Venous Access Device (TIVAD); Internal Jugular Vein; Chemotherapy; Colorectal Carcinoma

\section{Introduction}

Recently, significant improvements have been made in chemotherapy for colorectal carcinomas (CRCs). These improvements are mostly due to the development of combination chemotherapies including administration of fluorouracil, irinotecan, or oxaliplatin in conjunction with molecular targeting agents such as bevacizumab, cetuximab and panitumumab. Due to the efficacy of chemotherapy, its importance in the treatment of CRCs is on the rise. Notably, due to its ability to prolong overall survival, the duration of chemotherapy administration has been lengthened. In order to administer chemotherapeutic agents such as FOLFOX or FOLFIRI in outpatients, it is necessary to insert a Groshong catheter, which enables the patients to pull out the TIVAD needle by themselves. Administration of TIVAD avoids vascular pain that is caused during peripheral injection. Moreover, the peripheral injections occasionally cause extravasation injury caused by chemotherapeutic agents. Thus, TIVAD is a useful device to administer chemotherapy safely and with less damage.

The puncture site for insertion of the Groshong catheter of the TIVAD is through the subclavian vein (SV), 
internal jugular vein (IJV), femoral vein or axillary vein. In Japan, TIVADs are generally placed by the percutaneous subclavian vein puncture approach (SVPA). According to the Report from the Ministry of Health and Welfare in Japan (2009), about $82 \%$ of TIVADs were performed by SV puncture [1]. However, the perioperative complication rate of SVPA is reportedly higher than that of IJVPA.

The SV located just beneath the clavicle is punctured in SVPA. This approach has been shown to cause serious intraoperative or postoperative complications, such as pneumothorax, arterial puncture, hemothorax, injury of brachial plexus [2-6], and pinch-off syndrome [7]. When a misdirected puncture occurs to the subclavian artery, arterial hemostasis is very difficult to re-establish.

On the other hand, the IJV is located near the surface of skin, and it runs laterally to the carotid artery. IJV can be detected clearly by ultrasonography and is easily accessible. Thus, for TIVAD placement, the IJV approach is considered a low-stress procedure.

We examined the short-term outcomes of venous access via the IJV puncture approach for the placement of TIVADs compared with the SVPA.

\section{Patients and Methods}

Fifty-six consecutive patients who received chemotherapy for CRCs were enrolled in this study. They underwent TIVAD placement surgeries from June 2010 to August 2011 (Table 1). Written informed consent from each patient was obtained prior to the procedure. The choice of approach (IJVPA or SVPA) was adopted at the discretion of each doctor in charge of the patient.

Patients were brought to the X-ray fluoroscopy room and placed in a supine position. The operation was performed with local anesthesia ( $0.5 \%$ of Xylocaine, Astrazeneca, UK) and under maximal barrier precaution. The TIVAD placements were performed by two surgeons.

The operator punctured the IJV or SV under ultrasound guidance and inserted the guidewire using Seldinger's catheter technique. The catheter introducer was inserted into the vein along with the guidewire. The catheter was inserted through the introducer because the tip of the Groshong catheter (BardPort X-port isp, Bard Access Systems Inc., Salt Lake City, UT, USA) was closed to avoid blood reflux. The Groshong catheter was connected to the port, which was implanted into the subcutaneous space in the chest. The catheter tip position and shape of the catheter lumen were monitored by X-ray fluoroscopy. If the first IJV or SV puncture attempt was unsuccessful, the procedure was completed using the other approach. A chest radiography was performed after the operation to detect the position of the catheter tip and the distortion of the catheter lumen. Operation times, as well as intraoperative and postoperative complications were examined. Long-term complications were examined using an electronic medical chart. Computed tomography (CT) scans were performed to check for intravenous thrombosis and distortion of the catheter every $3-4$ months for 2 years and were evaluated by a radiologist and a surgeon. All patients were administered either FOLFOX or FOLFIRI and eighteen patients received simultaneous administration of bevacizumab.

\section{Results}

Fifty-six TIVAD placements were performed consecutively in total. IJVPA and SVPA were attempted in 30 and 26 cases, respectively. However, successful completion of IJVPA was obtained in 29 patients (Table 2) since it was necessary to convert to SVPA in 1 patient. SVPA was completed in only 23 patients since it was not successful in the remaining 3 patients and conversion to IJVPA was required. The completion rate was $96.5 \%$ in IJVPA and $88.5 \%$ in SVPA. Mean operation time was $34.3 \mathrm{~min}$ in IJVPA and $35.2 \mathrm{~min}$ in SVPA. No perioperative complications that required additional medical treatments were observed in both groups. In this study, no catheter distortion was detected by chest X-ray after the operation.

The total follow up period was 11,619 days in IJVPA and 11,563 days in SVPA. Late complications were observed in five cases, 3 by IJPVA and 2 by SVPA, which was not significantly different (Table 3). A catheterrelated thrombosis was found by $\mathrm{CT}$ scan in 3 patients, two of whom underwent IJVPA (6.7\%) and one case underwent SVPA (3.8\%). Two patients received simul-

Table 1. Comparison of patients.

\begin{tabular}{lccc}
\hline Patients & IJV & SV & p-value \\
\hline Number of Cases & 30 & 26 & n.s. \\
Gender (male/female) & 1.8 & 1.2 & n.s. \\
Age & 68.4 & 68.5 & n.s. \\
BMI & 20.7 & 21.6 & n.s. \\
\hline
\end{tabular}

Table 2. Early term results.

\begin{tabular}{lccc}
\hline Result & IJV & SV & p-value \\
\hline Mean operation time (min) & 34.3 & 35.2 & n.s. \\
Success rate \% & 96.5 & 88.5 & n.s. \\
Complication $^{*}$ & none & none & - \\
\hline
\end{tabular}

*No intraoperative or postoperative complications were observed. 
Table 3. Late complications.

\begin{tabular}{lccc}
\hline Complication & IJV & SV & p-value \\
\hline Catheter-related thrombosis & $2(6.7 \%)$ & $1(3.8 \%)$ & n.s. \\
Infection & $1(3.3 \%)$ & $1(3.8 \%)$ & n.s. \\
\hline
\end{tabular}

taneous administration of bevacizumab. Catheter infections occurred in 1 patient who underwent IJVPA (3.3\%) and 1 patient who underwent SVPA (3.8\%).

\section{Discussion}

The best approach for placement of TIVADs still remains unclear. In Japan, TIVADs have been generally placed by SVPA. However, the SVPA has been shown to cause intraoperative or postoperative complications including pneumothorax, arterial puncture, hemothorax, and injury to the brachial plexus [2-6]. The intraoperative complication rate of SVPA was reportedly higher than that of IJVPA [8-10].

Although the technical aspect of TIVAD placement has become much safer than before due to the use of image-guided navigation, like venous ultrasonography or venography, however, the risk of complications by mispuncture still remains. In particular, arterial bleeding from the subclavian artery by misdirected punctures can develop into very serious problems since this artery is located beneath the clavicle and vessel hemostasis is very difficult to re-establish [11].

Further, pinch-off syndrome has been reported as a complication of TIVAD which was placed by SVPA [7, 12-15]. This syndrome is thought to be caused by the compression of the catheter by the clavicle and the first rib [7,12], which may lead to obstruction followed by fracture of the catheter. Pinch-off syndrome is also referred to as subclavian crush syndrome in the cardiovascular field [16]. Subclavian crush syndrome has been reported to occur in some patients when pacemaker leads were implanted via SVPA. In these cases, conductor fracture and insulation breaches develop by compression of a lead that passes between the first rib and the clavicle. Once the catheter is torn, the remaining tip of the catheter is brought to the right atrium or pulmonary artery, which may lead to fatal consequences. A benefit of IJVPA is that this approach does not cause catheter compression that may lead to the pinch-off syndrome.

It remains controversial which approach of the two has higher rates of catheter-related thrombosis. Some previous reports have suggested a higher rate in IJVPA [17], although more recent reports have suggested otherwise, that IJVPA may indeed result in a lower risk of thrombosis $[8-10,18,19]$. In our study, no significant difference was observed in the rate of catheter-related thrombosis
Table 4. Catheter-related thrombosis with bevacizumab.

\begin{tabular}{ccccc}
\hline Complication & Bmab + & Bmab - & p-value & \\
\hline Thrombosis & + & $2(11.1 \%)$ & $1(2.6 \%)$ & \\
Thrombosis & - & 16 & 37 & n.s. \\
\hline
\end{tabular}

between IJVPA and SVPA. Bevacizumab has been reported to increase the risk of vein thrombosis in patients with TIVAD [20]. Although no statistically significant differences were found between the two groups in this study (Table 4), the incidence of thrombosis in the bevacizumab group (11.1\%) was higher than in the bevacizumab group (2.6\%). This finding suggests that catheter thrombosis could have been affected by administration of bevacizumab in our group of patients. Thus, clinicians should pay careful attention to the risk of catheter-related thrombosis in patients who receive administration of bevacizumab.

One further point to consider is the technical maneuver of IJVPA, which requires surgical technique to make a relatively long subcutaneous tunnel from the port to the puncture site. It is important to make a gradual curve of the subcutaneous tunnel to avoid creating a kink in the catheter.

\section{Conclusion}

In this study, we performed TIVAD placements by the IJVPA and evaluated the outcomes. We showed that IJVPA is a safe and feasible approach for TIVAD placement with outcomes that are comparable to the standard SVPA. Catheter thrombosis could have been affected by administration of bevacizumab.

\section{REFERENCES}

[1] Pharmaceuticals and Medical Devices Agency in Japan, 2009.

http://www.info.pmda.go.jp/kyoten_kiki/port.html\#umek omi

[2] R. Biffi, F. de Braud, F. Orsi, et al., "Totally Implantable Central Venous Access Ports for Long-Term Chemotherapy. A Prospective Study Analyzing Complications and Costs of 333 Devices with a Minimum Follow-Up of 180 Days," Annals of Oncology, Vol. 9, No. 7, 1998, pp. 767773. doi:10.1023/A:1008392423469

[3] E. H. Kincaid, P. W. Davis, M. C. Chang, J. M. Fenstermaker and T. C. Pennell, "'Blind' Placement of LongTerm Central Venous Access Devices: Report of 589 Consecutive Procedures," The American Journal of Surgery, Vol. 65, 1999, pp. 520-524.

[4] R. L. Poorter, F. N. Lauw, W. A. Bemelman, P. J. Bakker, C. W. Taat and C. H. Veenhof, "Complications of an Implantable Venous Access Device (Port-A-Cath) during 
Intermittent Continuous Infusion of Chemotherapy," European Journal of Cancer, Vol. 32A, No. 13, 1996, pp. 2262-2266. doi:10.1016/S0959-8049(96)00274-2

[5] C. E. Nightingale, A. Norman, D. Cunningham, J. Young, A. Webb and J. Filshie, "A Prospective Analysis of 949 Long-Term Central Venous Access Catheters for Ambulatory Chemotherapy in Patients with Gastrointestinal Malignancy," European Journal of Cancer, Vol. 33, No. 3, 1997, pp. 398-403. doi:10.1016/S0959-8049(97)89012-0

[6] I. Di Carlo, F. Barbagallo, A. Toro, M. Sofia, R. Lombardo and S. Cordio, "External Jugular Vein Cutdown Approach, as a Useful Alternative, Supports the Choice of the Cephalic Vein for Totally Implantable Access Device Placement," Annals of Surgical Oncology, Vol. 12, No. 7, 2005, pp. 1-4.

[7] D. A. Andris, E. A. Krzywda, W. Schulte, R. Ausman and E. J. Quebbeman, "Pinch-Off Syndrome: A Rare Etiology for Central Venous Catheter Occlusion," Journal of Parenteral and Enteral Nutrition, Vol. 18, No. 6, 1994, pp. 531-533. doi:10.1177/0148607194018006531

[8] C. Plumhans, A. H. Mahnken, C. Ocklenburg, S. Keil, F. F. Behrendt, R. W. Günther and F. Schoth, "Jugular versus Subclavian Totally Implantable Access Ports: Catheter Position, Complications and Intrainterventional Pain Perception," European Journal of Radiology, Vol. 79, No. 3, 2011, pp. 338-342. doi:10.1016/j.ejrad.2009.12.010

[9] R. C. Ribeiro, S. C. Abib, A. S. Aguiar and S. T. Schettini, "Long-Term Complications in Totally Implantable Venous Access Devices: Randomized Study Comparing Subclavian and Internal Jugular Vein Puncture," Pediatric Blood \& Cancer, Vol. 58, No. 2, 2012, pp. 274-277. doi: $10.1002 / \mathrm{pbc} .23220$

[10] C. Araújo, J. P. Silva, P. Antunes, J. M. Fernandes, C. Dias, H. Pereira, T. Dias and J. L. Fougo, "A Comparative Study between Two Central Veins for the Introduction of Totally Implantable Venous Access Devices in 1201 Cancer Patients," European Journal of Surgical Oncology, Vol. 34, No. 2, 2008, pp. 222-226. doi:10.1016/j.ejso.2007.04.003

[11] J. H. Lee, Y. B. Kim, M. K. Lee, J. I. Kim, J. Y. Lee, S. Y. Lee, E. J. Lee and Y. S. Lee, "Catastrophic Hemothorax on the Contralateral Side of the Insertion of an Implantable Subclavian Venous Access Device and the Ipsilateral Side of the Removal of the Infected Port-A Case Report," Korean Journal of Anesthesiology, Vol. 59, No. 3, 2010, pp. 214-219. doi:10.4097/kjae.2010.59.3.214

[12] D. H. Hinke, D. A. Zandt-Stastny, L. R. Goodman, E. J. Quebbeman, E. A. Krzywda and D. A. Andris, "Pinch-
Off Syndrome: A Complication of Implantable Subclavian Venous Access Devices," Radiology, Vol. 177, No. 2, 1990, pp. 353-356.

[13] R. C. Ribeiro, A. C. Monteiro, Q. C. Menezes, S. T. Schettini and S. M. Vianna, "Totally Implantable Catheter Embolism: Two Related Cases," Sao Paulo Medical Journal, Vol. 126, No. 6, 2008, pp. 347-349. doi:10.1590/S1516-31802008000600011

[14] A. E. Krutchen, H. Bjarnason, D. J. Stackhouse, G. K. Nazarian, J. E. Magney and D. W. Hunter, "The Mechanisms of Positional Dysfunction of Subclavian Venous Catheters," Radiology, Vol. 200, No. 1, 1996, pp. 159163.

[15] D. R. Aitken and J. P. Minton, “The 'Pinch-Off Sign': A Warning of Impending Problems with Permanent Subclavian Catheters," The American Journal of Surgery, Vol. 148, No. 5, 1984, pp. 633-636. doi:10.1016/0002-9610(84)90340-4

[16] M. Roelke, S. S. O’Nunain, S. Osswald, H. Garan, J. W. Harthorne and J. N. Ruskin, "Subclavian Crush Syndrome Complicating Transvenous Cardioverter Defibrillator Systems," Pace, Vol. 18, No. 5, 1995, pp. 973-979. doi:10.1111/j.1540-8159.1995.tb04737.x

[17] J. F. Timsit, J. C. Farkas, J. M. Boyer, J. B. Martin, B. Misset, B. Renaud and J. Carlet, "Central Vein Catheter-Related Thrombosis in Intensive Care Patients: Incidence, Risks Factors, and Relationship with CatheterRelated Sepsis," Chest, Vol. 114, No. 1, 1998, pp. 207213. doi:10.1378/chest.114.1.207

[18] S. Macdonald, A. J. Watt, D. McNally, R. D. Edwards and J. G. Moss, "Comparison of Technical Success and Outcome of Tunneled Catheters Inserted via the Jugular and Subclavian Approaches," Journal of Vascular and Interventional Radiology, Vol. 11, No. 2, 2000, pp. 225231. doi:10.1016/S1051-0443(07)61470-5

[19] A. Peris, G. Zagli, M. Bonizzoli, G. Cianchi, M. Ciapetti, R. Spina, V. Anichini, F. Lapi and S. Batacchi, "Implantation of 3951 Long-Term Central Venous Catheters: Performances, Risk Analysis, and Patient Comfort after U1trasound-Guidance Introduction," Anesthesia \& Analgesia, Vol. 111, No. 5, 2010, pp. 1194-1201. doi:10.1213/ANE.0b013e3181f333c1

[20] M. Yoshino, F. Kawahara, Y. Tanaka, M. Kaise, H. Nagai and Y. Takii, "A Study of Complications Associated with Central Venous Access Port Placement in Colorectal Cancer Patients Receiving Bevacizumab Chemotherapy," Journal of Japanese Society of Hospital Pharmacists, Vol. 48, 2012, pp. $307-311$. 